\title{
The Experience of Quality of Life for Caregivers of People With Alzheimer's Disease Living in Sardinia, Italy
}

\author{
Ercole Vellone, MSN, RN', Giovanni Piras, MSN, RN'2, Giulia Venturini, PhD, RN', \\ Rosaria Alvaro, MSN, RN', and Marlene Z. Cohen, PhD, RN, FAAN ${ }^{3}$
}

\begin{abstract}
Purpose:This study explored the meaning of quality of life (QOL) for Sardinian caregivers of people affected with Alzheimer's disease and factors improving and worsening their QOL. Design: The phenomenological method was used to study 4I Alzheimer's disease caregivers living on the western coast of Sardinia, Italy. Interviews were conducted and analyzed using Cohen, Kahn, and Steeves's approach. Findings: Extracted themes were the following: unity and cooperation in the family; freedom/independence; having time for themselves; serenity/tranquility; and well-being and health. Caregivers identified factors that they believed worsened or improved their QOL. Family was particularly important for these caregivers. Discussion and Practice Implications: This study reinforces previous research about cultural influence on QOL and emphasizes the importance of nurses being culturally sensitive. Since Sardinia is a rural region, these findings may be useful for nurses working with caregivers in other rural settings.
\end{abstract}

\section{Keywords}

caregivers, quality of life, Alzheimer's disease, Sardinia, Italy, phenomenological research, rural nursing, gerontology, transcultural health

\section{Introduction}

The prevalence of Alzheimer's disease (AD), the principal cause of dementia, is increasing worldwide as the population ages (Hall et al., 2009; Kalaria et al., 2008). Epidemiologic studies show that dementia after 65 years of age has a prevalence of $5 \%$ to $7 \%$ in all countries regardless of their stage of development (Hall et al., 2009; Kalaria et al., 2008). This includes Italy, where it is estimated that AD affects approximately 520,000 Italians (CENtro Studi Investimenti Sociali [CENSIS], 2007). Several Italian studies have focused on describing problems experienced by AD caregivers. These studies show that caregivers suffer from stress, burden, anxiety, depression, and sleep deprivation (Rinaldi et al., 2005; Sansoni, Vellone, \& Piras, 2004) and are overly involved in the patients' care (Vellone, Sansoni, \& Cohen, 2002).

Even though Sardinia is a part of Italy, it is an island $200 \mathrm{~km}$ off of Italy's western coast. So it has developed its own culture and social characteristics because of its geographical position and past domination (Berlinguer \& Mattone, 1998). Sardinia was dominated in the past by Phoenicians, Romans, Vandals, Byzantines, and Saracens, but one of the most powerful influence, which lasted for four centuries, was the domination by Spain that also influenced the language (Berlinguer \& Mattone, 1998). It has been discovered that even the genetic characteristics of Sardinians are different from other Italian people. In fact, about $42 \%$ of Sardinians are found to have the Y-DNA chromosome haplogroup I, which is frequently encountered in Northern and Eastern Europe but not in the rest of Italy (Calò, Melis, Vona, \& Piras, 2008).

Several scholars have studied the sociological and cultural aspects of Sardinia that make it different from the rest of Italy (Heatherington, 2006; Oppo, 1990, 1991; Sorge, 2007). Heatherington (2006) and Sorge (2007) described a cultural authenticity in Sardinians, where people feel a particular link to their land, family, and traditions that is rooted in social practices and expectations that are different from Italians or Europeans. Oppo (1990) found that Sardinian families are and live very close to each other, although they tend to be nuclear and not patriarchal (even in past) and this was different from the rest of Italy. She also found that Sardinian women, because of this past history of pastoral and agricultural duties that kept

\footnotetext{
'University of Rome "Tor Vergata," Rome, Italy

${ }^{2}$ Home Care Service, Oristano, Italy

${ }^{3}$ Nebraska Medical Center, Omaha, NE, USA

Corresponding Author:

Ercole Vellone, School of Nursing, University of Rome "Tor Vergata,"

Via Montpellier, I, 00133 , Rome, Italy

Email: ercole.vellone@uniroma2.it
} 
men far from the family, have had a symmetrical role with men because they were essential for financial and administrative contributions to the family (Oppo, 1991). According to the classification of the Organization for Economic Co-operation and Development [OECD] (2009), Sardinia is the Italian region with the most rural areas.

It has been observed that the above sociocultural aspects influence the caregiving of Sardinian older adults, which is primarily provided by women (Vellone \& Piras, 2009). Vellone and Piras (2009) found that Sardinian caregivers live at most 10 minutes by car from the older adults they look after. In addition, $90 \%$ of these caregivers declared that caring "makes the caregiver feel well" and 70\% said caring was an obligation. In another study (Vellone, Piras, Venturini, \& Alvaro, 2010), family support was found to explain most of the variance in the positive impact of caregiving to Sardinian older adults $(\beta=.71)$.

These aspects are uncommon in other parts of Italy and could change the way Sardinian caregivers perceive their quality of life (QOL). As emphasized by several authors (Kitrungroter \& Cohen, 2006; Scott et al., 2008), cultural and sociological aspects influence the way people perceive QOL. Kosberg, Kaufman, Burgio, Leeper, and Sun (2007) noted that rural caregivers of persons with dementia are an understudied population. Although they found differences between White and African American caregivers in rural Alabama based on their different culture, they also found similarities in the perceptions of tasks needed by care recipients, in health status, in social support services, and in QOL. Understanding caregivers from this rural setting may be useful in understanding caregivers in other rural areas whether or not they are from different cultures.

No data exist on AD in Sardinia but the assumption is that the epidemiology of this illness is similar to the rest of Italy. No studies have investigated the meaning of QOL for Sardinian AD caregivers. Although some international studies have shown that $\mathrm{AD}$ caregivers living in rural areas have worse QOL and use fewer formal services than those living in urban areas (Morgan, Semchuk, Stewart, \& D'Arcy, 2002; Tommis et al., 2007), no researchers have specifically explored the meaning of QOL for rural AD caregivers.

The purpose of this study was to describe the meaning of QOL for caregivers of Alzheimer patients living in Sardinia, and to discover factors that Sardinian caregivers believe worsen or improve their QOL. As emphasized by Campbell et al. (2008), QOL in caregivers of persons with dementia is more affected by their subjective interpretation and culture than by objective burden. Since Sardinia is a rural area, the experiences of AD caregivers living there may provide insights to those living in other rural areas of other countries.

\section{Background \\ Definitions of QOL}

Literature describes QOL as a multidimensional concept composed of subjective and objective constructs. Different definitions of QOL are reflected by the vast number of existing QOL instruments (Hendry \& McVittie, 2004). Despite the different definitions and conceptualizations of QOL, most authors consider QOL to be the combination of physical functioning, work, socioeconomic status, support network, housing, income, self-esteem, life satisfaction, lack of stress and burden, happiness, meaning of life, and health (Kaplan \& Ries, 2007; Kitrungroter \& Cohen, 2006). Some authors (S. C. Smith et al., 2005; Vellone, Piras, Talucci, \& Cohen, 2008) have also used qualitative methods to understand definitions of QOL for $\mathrm{AD}$ patients and caregivers. These studies have found that QOL for caregivers includes daily activities and looking after oneself, health and well-being, the care recipients' cognitive functioning, social relationships and self-concept, serenity, tranquility, freedom, and good financial status.

Informal caregivers are defined in the literature as relatives or friends who provide, without financial compensation, psychological and material support to another person needing care (CENSIS, 2007). They are an important source of care for people affected by $\mathrm{AD}$ in all countries because they provide patients with important support and allow patients to avoid institutionalization (Mittelman, Haley, Clay, \& Roth, 2006).

The care of patients with AD can profoundly affect the QOL of caregivers (Serrano-Aguilar, Lopez-Bastida, \& YanesLopez, 2006; Vellone et al., 2008), so a great deal of research has been carried out on this topic to find factors that can worsen or improve QOL (Vellone et al., 2008).

\section{Factors Worsening QOL in AD Caregivers}

Several researchers have found that QOL of AD caregivers is primarily worsened by psychobehavioral problems of patients (Ferrara et al., 2008). In addition, stress, depression, anxiety, burden, low income, and the severity and duration of $\mathrm{AD}$ also worsen caregivers' QOL (Andren \& Elmstahl, 2007; Ferrara et al., 2008). Caregivers' mental and physical health problems also worsen their QOL (Bruce et al., 2005). Predictors of poor QOL include caregiver overload, role capacity, confidence, neuroticism, previous relationship with the patients, the experience of caregiving (Campbell et al., 2008), and sleep disturbances (Creese, Bédard, Brazil, \& Chambers, 2008).

Studies focusing on identifying sociodemographic factors influencing QOL in AD caregivers have found that QOL is lower in depressed women and adult children caregivers (Andren \& Elmstahl, 2007), people less educated and older, and those spending more time in providing care (Argimon, Limon, Vila, \& Cabezas, 2004; Serrano-Aguilar et al., 2006). Researchers have also found that QOL in caregivers is affected by the cost of care, which increases with cognitive impairment and disease worsening (Leon \& Neumann, 1999).

In addition, ethnic differences have been found among caregivers for persons with dementia. Latina and African American AD caregivers have been found to be less stressed and anxious, to have better well-being, and to use fewer psychotropic medications than Caucasians (Coon et al., 2004; Haley et al., 2004). 
Haley et al. (1995) found that QOL was influenced more by ethnicity than by caregiving since White caregivers were more prone to have poorer QOL than were Black caregivers. Ethnic differences were also found in rural Alabama among caregivers for persons with dementia (Kosberg et al., 2007). Although similarities were found between White and African American caregivers related to perceptions of tasks needed by care recipients, health status, social support services, and QOL, White caregivers were more likely to be married and older, used acceptance and humor as coping styles, and had few financial problems whereas African American caregivers gave more hours of care, used religion and denial as coping styles, and were less burdened (Kosberg et al., 2007).

Qualitative research has examined factors worsening QOL of AD caregivers but has not specifically or primarily focused on this topic. Morgan et al. (2002) used focus groups to study barriers to use of formal services. These formal services have been found to improve QOL for caregivers. These barriers were the stigma of dementia, lack of privacy, beliefs and attitudes, lack of awareness, financial barriers, acceptability of services, and challenges in service delivery. Another qualitative study used in-depth interviews and revealed that AD caregivers experienced loss, sadness, compromised life satisfaction, and poor psychological health when they relinquished full-time care and patients are institutionalized (Chene, 2006). Vellone et al. (2008) found in a phenomenological study that worries about the future, progression of the patient's illness, and stress all worsened QOL in AD caregivers.

\section{Factors Improving QOL in AD Caregivers}

Much research has focused on finding factors that improve QOL in AD caregivers. This literature is based mainly on intervention studies testing several methods to improve QOL in caregivers. Factors shown to improve caregiver QOL and decrease patients' institutionalization include providing support of a care manager (Kuroda et al., 2007), providing caregivers with information, role playing, problem solving, skill training, occupational activities, and stress management (Belle et al., 2006; Chien \& Lee, 2008). Caregiver burden has been reduced and QOL improved through interdisciplinary psychoeducational family group interventions (Ostwald, Hepburn, Caron, Burns, \& Mantell, 1999) and by respite services where patients can be temporarily cared for, allowing caregivers some rest from caring (Jones \& Peters, 1992).

Qualitative research has not always focused on describing factors improving QOL in AD caregivers. B. Smith, ChurHansen, Neale, and Symon (2008), in a study with open interviews about the effects of cholinesterase inhibitors on $\mathrm{AD}$ patients, found that this treatment did not improve the emotional QOL in patients and in their caregivers. However, a study using focus groups found that, compared with standard home care, a specialist multiagency home support service reduced stress in caregivers (Rothera et al., 2008). Vellone et al. (2008) found that factors improving the $\mathrm{QOL}$ in $\mathrm{AD}$ caregivers were the good health of the patient, independence from the patient, and more help in caregiving.

Studies in the literature have primarily used a quantitative approach to find variables that significantly worsen or improve QOL in AD caregivers. Little attention has been given to the meaning of QOL for the caregivers themselves although QOL is considered the principal outcome of all health care interventions. In addition, the experience of QOL for rural AD caregivers is understudied. It is also important to study particular cultures (such as Sardinia) since culture has been found to influence QOL.

\section{Method \\ Design}

This study was guided by the phenomenological method (Cohen, Kahn, \& Steeves, 2000). This approach has been used by Cohen, Pace, Kaur, and Bruera (2009), and was modified by Vellone et al. (2002) and Vellone et al. (2008) to involve researchers from different languages. This method included the following activities: (a) critical reflection before data collection, which required researchers to write their beliefs and assumptions on the phenomenon to ensure bracketing during data analysis; (b) interview of participants with open-ended questions until data saturation, recording all interviews and taking field notes to reconstruct the environments and settings, to report body language and tone of voice, and to note researchers' reflections; (c) verbatim transcription of interviews and checking for their accuracy. (In the present study, one of the authors [GP] transcribed the interviews and then another one [EV] listened to the interviews and checked the accuracy of the transcripts.); (d) translation of transcripts and field notes into the foreign language (English in this study) by a bilingual teacher and the back-translation of the same transcripts into the original language by another bilingual teacher; (e) comparison between the translated and the back-translated transcripts and field notes to ensure that the version to be sent to the foreign investigator was accurate; (f) immersion in the data by reading and rereading the transcripts and the field notes repeatedly; (g) extraction of themes by all researchers with a reflective awareness that leads to a dialectical examination of parts of the data to better understand the whole and vice versa (i.e., the hermeneutic circle - the extraction of themes by foreign researchers is done on transcripts and field notes in their language after the translation and back-translation process); (h) establishment of theme trustworthiness, with a comparison of themes extracted by each researcher (themes extracted by the foreign researcher undergo translation and back-translation before comparison), discussion among researchers to reach an agreement (discussion with the foreign investigator was done by e-mail and phone), and the consultation of the written text made with the critical reflection technique; (i) presentation of extracted themes to study participants for their confirmation; and (j) writing the scientific report. 
Journal of Transcultural Nursing $X X(X)$

\section{Study Participants}

Participants were 41 primary caregivers of people with AD. Table 1 reports sociodemographic data. Most participants were female, nearly 60 years old, married, not working, and cared for patients almost 17 hours per day on average. The majority of caregivers were living with the patients and had been caring for them for more than 4 years. Participants had a little more than 9 hours of free time per week.

Most AD patients were female, were older than 75 years, and only nine of them had a score of 1 or 2 (mild and moderate dementia) on the Clinical Dementia Rating Scale (Hughes, Ber, \& Danziger, 1982). A total of 26 patients were treated with acetylcholinesterase inhibitors.

\section{Data Collection and Analysis}

Ethical approval was obtained before the study began. In all, 28 caregivers were recruited from the Alzheimer's Association on the western coast of Sardinia, Italy; the rest were enrolled with a snowball procedure from the same area. Only primary caregivers who cared for AD patients for at least 2 years were asked to take part in the study. Study aims were explained and participants signed informed consent. Participants were interviewed in Italy in their own homes without the patient. During the interviews, some patients were sleeping in bed and others were entertained in another room by another family member. Questions asked of the caregivers included the following:

1. Considering the fact that you take care of a person affected by AD, what do you think QOL is?

2. Which factors do you think improve your QOL?

3. Which factors do you think worsen your QOL?

Interviewers were welcoming and reassuring during the interview to facilitate caregivers describing their experiences. When caregivers stopped talking, interviewers asked if they had anything more to add. These questions have been used by the research team in a prior study (Vellone et al., 2008) and they allowed participants to describe their experiences of QOL. Participants gave examples of concepts that were important to their QOL.

Data saturation (no new themes emerged from the participants' experiences) was achieved with $41 \mathrm{AD}$ caregivers. Interviews lasted 30 minutes on average and were done only once. However, after the extraction of themes, researchers met participants a second time to show them the extracted themes and get their approval. All caregivers verified that the extracted themes accurately reflected their experiences.

\section{Findings}

Themes from the analysis of transcripts and field notes were categorized according to the three questions posed to the caregivers (Table 2). Participants reported many experiences related
Table I. Participants' Sociodemographic Data $(N=4 I)$

\begin{tabular}{|c|c|c|c|c|}
\hline & Mean & $S D$ & Range & $N(\%)$ \\
\hline \multicolumn{5}{|l|}{ Gender } \\
\hline Male & & & & $6(14.6)$ \\
\hline Female & & & & $35(85.4)$ \\
\hline Age (years) & 59.20 & 11.82 & $26-78$ & \\
\hline \multicolumn{5}{|l|}{ Education } \\
\hline Elementary & & & & $3(7.3)$ \\
\hline Middle & & & & $13(31.7)$ \\
\hline High & & & & $16(39)$ \\
\hline Degree & & & & $9(22)$ \\
\hline \multicolumn{5}{|l|}{ Marital status } \\
\hline Married & & & & $32(78)$ \\
\hline Single & & & & $8(19.5)$ \\
\hline Widowed & & & & I (2.4) \\
\hline \multicolumn{5}{|l|}{ Profession } \\
\hline Employed & & & & $12(29.27)$ \\
\hline Unemployed & & & & $29(70.73)$ \\
\hline $\begin{array}{l}\text { Caregiver living with } \\
\text { patient }\end{array}$ & & & & $33(80.5)$ \\
\hline $\begin{array}{l}\text { Time since diagnosis } \\
\text { (months) }\end{array}$ & 54.15 & 23.57 & $24-130$ & \\
\hline $\begin{array}{l}\text { Free time of caregivers } \\
\text { per week (hours) }\end{array}$ & 9.34 & 9.35 & $2-60$ & \\
\hline Hour of care per day & 16.93 & 5.71 & $3-24$ & \\
\hline Age of patients (years) & 76.54 & 8.19 & $57-90$ & \\
\hline $\begin{array}{l}\text { Clinical Dementia } \\
\text { Rating Scale score }\end{array}$ & 3.13 & 1.04 & $1-5$ & \\
\hline \multicolumn{5}{|l|}{ Gender of patients } \\
\hline Male & & & & $12(29.3)$ \\
\hline Female & & & & $29(70.7)$ \\
\hline $\begin{array}{l}\text { Acetylcholinesterase } \\
\text { treatment }\end{array}$ & & & & $26(63.4)$ \\
\hline \multicolumn{5}{|l|}{$\begin{array}{l}\text { Relationship with } \\
\text { patient }\end{array}$} \\
\hline Spouse & & & & $13(19.5)$ \\
\hline Adult child & & & & $26(63.4)$ \\
\hline Friend & & & & $2(4.9)$ \\
\hline
\end{tabular}

a. Clinical Dementia Rating Scale score: The higher the score, the worse the dementia degree (range of scores is 0-5).

to QOL and what factors, in their experiences, improved or worsened them. Many of these experiences overlapped when examining responses to the three questions. In addition, some themes extracted from responses to a question were similar to themes from another question. For example, "family" was in both what QOL meant for caregivers and in factors improving QOL.

\section{What is QOL}

Four themes were extracted from the question asking caregivers to describe the meaning they attributed to QOL in their situations: 
Table 2. Extracted Themes From Phenomenological Analysis

What QOL is
Unity and cooperation in the family
Freedom, independence, having time for themselves
Serenity/tranquility
Well-being and health
Factors worsening QOL
Fear for the future: For the care needed and for the illness
worsening
Continuous care of the patients, not having time for themselves
Factors improving QOL
No worsening of the illness
Help and support from family
Help from formal services
Satisfaction and reward from giving care
Financial support for paying other assistants
More free time
More public sensitization about AD

Note: $A D=$ Alzheimer's disease; $\mathrm{QOL}=$ quality of life.

Unity and cooperation in the family. The word "family" recurred many times during the interviews, making it a central concept in the caregivers' meaning of QOL. Almost all caregivers thought their QOL was linked to the unity and cooperation in the family. Emblematic are the following sentences from two caregivers: "For me QOL is unity and cooperation in the family [ ... ] if I would not have my family so united and all their collaboration, how could I face the everyday problems?" "My mother's illness totally modified my QOL, which was good in the past. Today she needs total care and attention from me and all the family. My QOL isn't poor because I have all the support from my family." This interview was conducted with the caregiver sitting in her living room. She frequently looked at pictures of family members during the interview.

Freedom, independence, having time for themselves. The caregivers in this study gave a great deal of care to their patients. More than $80 \%$ of those involved in this study lived with the patients, gave care for almost 17 hours per day, and had, on average, only 9 hours of free time per week. Many caregivers discussed not having freedom or independence because they had to care for the patients for so much of the day. A caregiver, who was the son of an $\mathrm{AD}$ patient, stated,

Quality of life means to live in freedom [ ... ]. With Alzheimer's this is not possible because you are compelled to depend on the needs of another person and you cannot carve a little time essential for your own life.

Another son stated, "QOL means to be free to do whatever you like without depending on anybody."

Serenity/tranquility. Many caregivers used the terms serenity and tranquility to define QOL during the interview. A wife said, "To have a good QOL means to be serene and tranquil, in peace with oneself and others. It is four years that I'm not serene and tranquil anymore." A daughter, before expressing her view about QOL, said, "All the problems caused by AD suffocate you! Since my mother had Alzheimer's I'm not serene and tranquil anymore with myself and others." This daughter started crying after making this statement.

Well-being and health. Well-being and health were cited by many caregivers when talking about the meaning of QOL. Many emphasized that they did not have well-being or health. A wife reported, "QOL is when you have wellbeing [ ... ]: you have enough money, you are well psychologically, you are in good health. I had well-being until 3 years ago, before my husband's illness. Now I am unwell!" Another wife specifically defined QOL using the term health:

If you have money but don't have health you can't have a good QOL. Since my husband has Alzheimer's I have many health problems and the paradox is that as I feel worse and worse I have less and less time to go to the doctor and care for myself.

\section{Factors Worsening QOL}

Two themes emerged from factors that participants thought worsened their QOL.

Fear for the future: For the care needed and for the illness worsening. Almost all caregivers feared what would happen in the future. They discussed several kinds of fear: of not being able to afford care expenses, that the patient would be bedridden "like a vegetable" with pressure ulcers, of leaving the patient, of getting physically and psychologically exhausted, of dying before the patient with concerns about with whom to leave the patient, and of not having the ability to manage the situation in the future. A husband said,

Until now I succeed in managing the situations related to my wife's illness even if I am alone. But I'm afraid about the future. I'm afraid the illness will worsen and I could not care for my wife as I do now.

A daughter reported, "Things that worsen my QOL are the fear that the illness can degenerate and I can't see my mother living in a bed like a vegetable." Another daughter said, "I am afraid she can be confined in bed and I don't want her to have bedsores that will cause her further suffering."

Continuous care of the patients: Not having time for themselves. Participants very often noted during the interviews that they had to care for their patients too many hours a day. More than $63 \%$ of these caregivers were the adult children of the patients, most were daughters, and none received help from the National Health Service. These caregivers also had their own families to care for, so they felt overwhelmed. A daughter, appearing stressed and anxious, stated,

My mother lives with me and I continuously take care of her. I also have to have her under control to see if she 
does something dangerous - sometimes a neighbour calls me and tells me that my mother is leaning out of the window. So, I never have a minute for me.

Another daughter reported,

My life is worsened by the fact that I have to care for my father for too many hours a day. When I come home, after assisting him, my job is not yet finished: I have a husband and two children!

Part of this interview was done while the participant was cooking. She apologized for doing this during the interview but said she had no time to stop and speak while just sitting.

\section{Factors Improving QOL}

Caregivers were also asked about factors that could have improved their QOL in their specific situations. Extracted themes are as follows:

No worsening of the illness. All caregivers were aware of illness progression but despite this they hoped the illness would not worsen. A son stated, "I feel my QOL could improve if I had certainty the illness wouldn't worsen. But I also know that it is not possible to stop Alzheimer's." This participant looked resigned while saying this. A husband said, "I would live really well if my wife's health wouldn't worsen: I am a cardiac patient and can't stand the idea that tomorrow she can't rely on me."

Help and support from family. A central reference for these caregivers was family and was a component of one theme in the meaning that participants gave to QOL (unity and cooperation in the family). Caregivers perceived the help and support given by family as a factor improving their QOL. A daughter noted, "An element that improves my QOL is my family. I receive great help from my husband. Even if he doesn't give me material help, his presence is fundamental in the moments of discouragement and sadness when some tears fall."

Some spouses recognized the importance of their family in improving their QOL but tended to not excessively involve family members in caregiving as reported by a wife: "The presence of my children is a point of strength in improving my QOL [ ... ] but I tend to not burden them with my thoughts, anguishes, and worries because I want them to live in serenity." A daughter said,

What improves my QOL is the sustenance of my family, not only my children and my husband, but also my family of origin. Everyone gives their best in providing care for my mother $[\ldots]$ and this gives me the courage to go on.

Help from formal services. The majority of caregivers said that their QOL would be improved if they received more help from formal services. Unfortunately, none of these caregivers received help from the National Health Service and many of them, particularly those with no other family members to help, could not afford to pay a person to help them assist the patient. Many participants expressed anger when talking about the lack of help they received. A husband stated,

What would help me to live a better QOL would be the help from other people in order to help me in caring for my wife. It would be only to give her some company, to talk with her while I could have a little time to myself, even an hour a day.

A wife said, "My QOL would improve a lot with help from other people and from the National Health Service."

Satisfaction and reward from giving care. Almost all participants felt satisfaction and reward from caregiving, which they noted improved their QOL. These feelings were visible on participants' faces. Sometimes caregivers cried or looked angry while reporting their experiences, but when they talked about the satisfaction and reward for giving care they immediately changed their facial expression. Despite the stress caused by caregiving, almost all participants felt they were doing the "best thing" for the patient and were happy when seeing the patient serene because of the care they received. Caregivers also considered the caregiving a duty in their role as child or spouse. A daughter reported, "What improves the quality of my life is the sense of reward when I see my mother happy and serene for the care I can give her." A son said,

Despite the problem of having my mother with Alzheimer, I get satisfaction when I see her serene [ . . . ]. When I see she is happy for how my brother, my father and I care for her, this is a reward for me as a child.

Financial support for paying for assistance. Some caregivers felt very stressed by the care they gave to the patient. They needed material help (not just psychological support) in providing care. They could have benefited from financial support so that they could pay an assistant with whom to divide care duties. A wife expressed anger in her body language and said,

My condition will improve with better financial support. Since my husband got sick he could not work anymore and so our financial income has decreased. The little money from the pension is used to pay for medicines and other medical things for my husband. With more money I could afford to pay other people who can help me to care for my husband.

A daughter reported similar problems: "I need better financial support. With more money I could pay other people who can assist my mother while I could go do shopping or walking."

More free time. Lack of time was a problem for the participants of this study, who had little or no time for themselves. More "free time" was a factor caregivers identified as improving QOL. A daughter said, "To have more time for me would improve my QOL. I would like to dedicate time to my hobbies. 
Before my mother got sick I used to go swimming. I am athletic and I miss this activity." In this participant's living room were two pictures of her swimming and she pointed to them with melancholy while speaking about this old hobby.

More public sensitization about AD. The majority of caregivers noted that most people lack knowledge about Alzheimer's. They felt isolated, not understood by others, and abandoned. Some of these participants appeared sad and quite resigned to their situations, but some were particularly agitated and angry when discussing this. Caregivers said they would have better QOL if people were more sensitive to, and more educated about, the problems they experience. A wife reported, "It would be better if people were more sensitive to Alzheimer's. Very often I see attitudes that show that people don't understand my hardship. I would like to be more understood by people." This wife raised her voice while saying this last sentence.

A daughter said, "Few people can understand my condition. There is so much ignorance! Some people think that I help my father for my interest. They think Alzheimer's is an illness that produces money. It's absurd!"

\section{Discussion}

This study is the first carried out in the rural area of Sardinia with the aim of describing the experience of QOL for AD caregivers and factors they consider as worsening and improving their QOL. Few studies of caregivers of persons with dementia have used a qualitative approach to specifically examine the meaning of QOL and factors improving or worsening QOL for AD caregivers (Butcher, Holkup, \& Buckwalter, 2001; Vellone et al., 2008). The experience of QOL, in this sample, reflects both aspects already reported related to the general definition of QOL (Kaplan \& Ries, 2007; Kitrungroter $\&$ Cohen, 2006) and new aspects not found in the literature. A new idea about QOL that caregivers in this study emphasized was that QOL, in their view, meant "unity and cooperation in the family." Family and social support for AD caregivers have been shown to be important to them in several studies and to improve QOL (Vellone et al., 2002; Wilks \& Croom, 2008). In this study, help and support from the family were found to be factors that improved caregivers' QOL and this reinforces what was found in a previous study carried out with Sardinian caregivers of older adults in which the support from family was a predictor of the positive impact of caregiving (Vellone et al., 2010). However, when participants were asked about what QOL is, they referred to the specific aspects of "family," "unity" and "cooperation." These aspects are original, in that no prior studies have found that the QOL of AD caregivers means unity and cooperation in family. This finding may reflect the cultural orientation of Sardinians that emphasizes the importance of family presence in their life (Oppo, 1990, 1991).

The other themes that described the meaning of QOL for the participants were similar to those found in a study carried out with caregivers living in Rome, Italy (Vellone et al., 2008). In that study, freedom/independence, serenity/tranquility, and well-being were central to the meaning of QOL for caregivers. In contrast, QOL for Sardinian caregivers included independence but not good financial status. Actually, independence was also a theme used in the sample from Rome (Vellone et al., 2008) but as a factor improving the caregiver's QOL. Similarly "good financial status" was used by participants from Rome to define QOL whereas Sardinian caregivers saw it as a factor improving QOL. The theme freedom, independence, and having time for themselves, which emerged in the present study, reinforces prior research in Rome that found that the deep involvement in care that caregivers experience also resulted in a reduction in caregivers' well-being and health (Spadin, 2008).

The caregivers in this study discussed fear for the future, the continuous care of the patient, and having no time for themselves as factors worsening their QOL. The future also was a concern for Roman caregivers but they felt only "worries" about it. Instead, when Sardinian participants reported factors worsening QOL, they specifically used the noun "fear" or the verb "to be afraid" and not just "worries." Their fears for the future were related to concern about providing care and about the illness worsening. These themes reflect the excessive involvement and the burden of caregivers as demonstrated in many studies (Andren \& Elmstahl, 2007; Campbell et al., 2008; CENSIS, 2007). To our knowledge no other research has found fear about the future in AD caregivers. In the study by Nichols (2001), fear was reported but referred to dealing with uncertainty and aloneness. Warner (1999) did not find fear about caregiving in the future.

In contrast to other studies (e.g., Vellone et al., 2008), very few Sardinian caregivers used the word "stress" when talking about factors worsening their QOL. When they referred to what might be considered stress, they were specific in describing stressful experiences; they meant the continuous care of patients and not having time for themselves. Several authors have found, with quantitative methodologies that excessive involvement in caregiving can reduce the time caregivers have for themselves and consequently their QOL (Andren \& Elmstahl, 2007; Campbell et al., 2008; Ferrara et al, 2008; Spadin, 2008). The findings of the present study corroborate these experiences of caregivers in prior research.

Factors improving QOL in Sardinian caregivers showed interesting themes not found in the literature: "help and support from family," "satisfaction and reward from giving care," and "more public sensitization about AD." The first theme is similar to the one that caregivers used to define QOL, which reinforced the importance of family for Sardinians. "Satisfaction and reward" was another frequent theme in caregivers' experiences and also can be connected with the deep sense of family for these caregivers. Prior research has reported that giving care to AD patients is a stressful experience but sometimes produces satisfaction and reward (Andren \& Elmstahl, 2005). Prior research with Sardinian caregivers of older adults showed that $90 \%$ of them considered caregiving as a positive experience (Vellone \& Piras, 2009). Heru and Ryan (2006) found that reward was negatively correlated with strain and 
burden in caregivers and positively correlated with higher family function, which is similar to the present study. Sanders (2005) also found rewards in AD caregivers who gained in spiritual growth, personal growth, and feelings of mastery. Satisfaction was also found in many studies of AD caregivers (Arai, Arai, \& Zarit, 2008; Habermann \& Davis, 2005) and was found to be positively correlated with a satisfactory relationship before the illness and quality of family functioning, and negatively correlated with burden (Andren \& Elmstahl, 2005; Steadman, Tremont, \& Davis, 2007). The theme "more public sensitization about AD" was extracted from many interviews. Caregivers felt lack of understanding and abandonment by "normal" people. This theme might be related to caregivers living in rural areas receiving less support and services and less information on behavioral problems of patients (Edelman, Kuhn, Fulton, \& Kyrouac, 2006; Morgan et al., 2002). Studies have found that the general population has little knowledge of AD (Arai et al., 2008; Rimmer, Wojciechowska, Stave, Sganga, \& O'Connell, 2005) and this can contribute to misunderstandings of problems faced by these families.

All these interviews were conducted in the caregivers' homes (i.e., in a natural setting). This helped caregivers be open in describing their experiences. Although phenomenological research should be carried out in the natural setting of the participants, no studies on AD caregivers have done this in Italy for reasons connected with participants' privacy and other practical obstacles. Sardinians are a hospitable and welcoming people who enjoyed being interviewed in their homes.

\section{Limitations}

One limitation of the study, as for all research with a qualitative methodology, is that the results are not generalizable. However, some themes identified in the present study were also reported in the literature (Vellone et al., 2008; Vellone et al., 2010). Another limitation is that more than two thirds of the sample was affiliated with an Alzheimer's Association, which might influence perceptions and feelings of participants about QOL. However, themes from those affiliated with the Alzheimer's Association were similar to those of other participants.

We used three structured questions for this study that might have limited participants fully reporting their lived experiences of QOL related to AD caregiving. These questions however helped caregivers verbalize and focus on the research topic. In fact, previous Italian studies have shown that caregivers of persons with dementia live with many problems and experiences that require more narrowed studies (CENSIS, 2007; Vellone et al., 2002). In any case, although interview questions were structured, the answers were open and caregivers freely reported whatever they felt during the interview. They did describe their experiences of caregiving. Although generalizability is not a goal for qualitative research, we acknowledge that our study of AD caregivers living in Sardinia may not apply to others outside this area. However, the meaning of QOL for these caregivers may also apply to others living in rural areas.

\section{Implications}

Findings in this study reinforced previous research on cultural influences on QOL (Kitrungroter \& Cohen, 2006; Scott et al., 2008) and emphasized the importance of health care professionals being sensitive to both culture and context (i.e., difference for those in rural settings). As Whitehouse, Gaines, Lindstrom, and Graham (2005) highlighted, the cultural competence of health care professionals may produce improvements in dementia care, elucidating sociocultural processes and cultural beliefs that help in better understanding the experience of illness and caregiving. These findings could be useful to Sardinian health care professionals in becoming more sensitive to the needs of caregivers, which is a primary use of the qualitative approach (Cohen et al., 2000). Since Sardinia is a rural setting, these findings may also apply to other rural caregivers in other countries.

QOL is a principal outcome of health interventions. The findings in this study could help health care professionals better understand QOL assessments and implement more effective interventions for $\mathrm{AD}$ caregivers. QOL was defined as unity and cooperation in the family. Health and support from family also was identified as a factor improving QOL. Although family is always involved in the care of $\mathrm{AD}$ patients in all countries, Sardinian health care professionals and those working in rural contexts should emphasize the importance of family in the care of AD patients. Since reward and satisfaction for the care given was considered to improve QOL, health care professionals should bolster these feelings in caregivers since caregivers with higher satisfaction and reward have better QOL (Heru \& Ryan, 2006). Sardinian nurses should sensitize politicians to provide more resources for caregivers. It has been shown that rural caregivers have worse QOL and fewer services than urban caregivers (Tommis et al., 2007) and could benefit from supportive programs (S. A. Smith \& Bell, 2005). Public sensitization about $\mathrm{AD}$ was identified as critical by participants in this study. A media information campaign might help $\mathrm{AD}$ caregivers feel better understood and less abandoned. The fear about the future that these caregivers felt perhaps could be reduced by helping them establish short-term objectives in care and by supporting them with educational interventions that have proven effective in reducing burden and stress and in improving QOL (Belle et al., 2006; S. A. Smith \& Bell, 2005).

\section{Declaration of Conflicting Interests}

The author(s) declared no potential conflicts of interest with respect to the research, authorship, and/or publication of this article.

\section{Funding}

The author(s) received no financial support for the research, authorship, and/or publication of this article.

\section{References}

Andren, S., \& Elmstahl, S. (2005). Family caregivers' subjective experiences of satisfaction in dementia care: Aspects of burden, 
subjective health and sense of coherence. Scandinavian Journal of Caring Sciences, 19, 157-168.

Andren, S., \& Elmstahl, S. (2007). Relationships between income, subjective health and caregiver burden in caregivers of people with dementia in group living care: A cross-sectional community-based study. International Journal of Nursing Studies, 44, 435-446.

Arai, Y., Arai, A., \& Zarit, S. H. (2008). What do we know about dementia? A survey on knowledge about dementia in the general public of Japan. International Journal of Geriatric Psychiatry, 23, 433-438.

Argimon, J. M., Limon, E., Vila, J., \& Cabezas, C. (2004). Healthrelated quality of life in carers of patients with dementia. Family Practice, 21, 454-457.

Belle, S. H., Burgio, L., Burns, R., Coon, D., Czaja, S. J., GallagherThompson, D., . . Z Zhang, S. (2006). Enhancing the quality of life of dementia caregivers from different ethnic or racial groups: A randomized, controlled trial. Annals of Internal Medicine, 145, 727-738.

Berlinguer, L., \& Mattone, A. (1998). La Sardegna [Sardinia]. Turin, Italy: Einaudi Editore.

Bruce, D. G., Paley, G. A., Nichols, P., Roberts, D., Underwood, P. J., ... Schaper, F. (2005). Physical disability contributes to caregiver stress in dementia caregivers. The Journals of Gerontology. Series A: Biological Sciences and Medical Sciences, 60, 345-349.

Butcher, H., Holkup, P., \& Buckwalter, K. (2001). The experience of caring for a family member with Alzheimer's disease. Western Journal of Nursing Research, 23, 33-55.

Calò, C. M., Melis, A., Vona, G., \& Piras, I. S. (2008). Sardinian population (Italy): A genetic review. International Journal of Modern Anthropology, 1, 39-64.

Campbell, P., Wright, J., Oyebode, J., Job, D., Crome, P., Bentham, P., . . Lendon, C. (2008). Determinats of burden in those who care for someone with dementia. International Journal of Geriatric Psychiatry, 23, 1078-1085.

CENtro Studi Investimenti Sociali. (2007). I costi economici e sociali della malattia di Alzheimer: Cosa è cambiato? [Economical and social costs of Alzheimer Disease: What has changed?]. Rome, Italy: Author.

Chene, B. (2006). Dementia and residential placement: A view from the carers' perspective. Qualitative Social Work, 5, 187-215.

Chien, W. T., \& Lee, Y. M. (2008). A disease management program for families of persons in Hong Kong with dementia. Psychiatric Services, 59, 433-436.

Cohen, M. Z., Kahn, D., \& Steeves, R. (2000). Hermeneutic phenomenological research: A practical guide for nurse researchers. Thousand Oaks, CA: Sage.

Cohen, M. Z., Pace, E. A., Kaur, G., \& Bruera, E. (2009). Delirium in advanced cancer leading to distress in patients and family caregivers. Journal of Palliative Care, 25, 164-171.

Coon, D. W., Rubert, M., Solano, N., Mausbach, B., Kraemer, H., Arguëlles, T., .. . Gallagher-Thompson, D. (2004). Well-being, appraisal, and coping in Latina and Caucasian female dementia caregivers: Findings from the REACH study. Aging \& Mental Health, 8, 330-345.
Creese, J., Bédard, M., Brazil, K., \& Chambers, L. (2008). Sleep disturbances in spousal caregivers of individuals with Alzheimer's disease. International Psychogeriatric, 20, 149-161.

Edelman, P., Kuhn, D., Fulton, B. R., \& Kyrouac, G. A. (2006). Information and service needs of persons with Alzheimer's disease and their family caregivers living in rural communities. American Journal of Alzheimer's Disease \& Other Dementias, 21, 226-233.

Ferrara, M., Langiano, E., Di Brango, T., De Vito, E., Di Cioccio, L., \& Bauco, C. (2008). Prevalence of stress, anxiety and depression in with Alzheimer caregivers. Health \& Quality of Life Outcomes, $6,1-5$.

Habermann, B., \& Davis, L. (2005). Caring for family with Alzheimer's disease and Parkinson's disease: Needs, challenges and satisfaction. Journal of Gerontological Nursing, 31, 49-54.

Haley, W. E., Gitlin, L. N., Wisniewski, S. R., Mahoney, D. F., Coon, D. W., Winter, L., . . . Ory, M. (2004). Well-being, appraisal, and coping in African-American and Caucasian dementia caregivers: Findings from the REACH study. Aging \& Mental Health, 8, 316-329.

Haley, W. E., West, C. A., Wadley, V. G., Ford, G. R., White, F. A., Barrett, J. J., . . Roth, D. L. (1995). Psychological, social, and health impact of caregiving: A comparison of Black and White dementia family caregivers and noncaregivers. Psychology and Aging, 10, 540-552.

Hall, K., Gao, S., Baiyewu, O., Lane, K. A., Gureje, O., Shen, J., . . Hendrie, H. C. (2009). Prevalence rates for dementia and Alzheimer's disease in African Americans: 1992 versus 2001. Alzheimer's \& Dementia, 5, 227-233.

Heatherington, T. (2006). Sin, saint, and sheep in Sardinia. Identities, 13, 533-556.

Hendry, F., \& McVittie, C. (2004). Is quality of life a healthy concept? Measuring and understanding life experiences of older people. Qualitative Health Research, 14, 961-975.

Heru, A. M., \& Ryan, C. E. (2006). Family functioning in the caregivers of patients with dementia: One-year follow-up. Bulletin of Menninger Clinic, 70, 222-231.

Hughes, C. P., Ber, L., \& Danziger, W. L. (1982). A new scale for the staging of dementia. British Journal of Psychiatry, 140, 566-572.

Jones, D. A., \& Peters, T. J. (1992). Caring for elderly dependents: Effects on carers' quality of life. Age and Ageing, 21, 421-428.

Kalaria, R., Maestre, G. E., Arizaga, R., Friedland, R. P., Galasko, D., Hall, K., . . . Antuono, P. (2008). Alzheimer's disease and vascular dementia in developing countries: Prevalence, management, and risk factors. Lancet Neurology, 7, 812-826.

Kaplan, R. M., \& Ries, A. L. (2007). Quality of life: Concept and definition. COPD: Journal of Chronic Obstructive Pulmonary Disease, 4, 263-271.

Kitrungroter, L., \& Cohen, M. Z. (2006). Quality of life of family caregivers of patients with cancer: A literature review. Oncology Nursing Forum, 33, 625-632.

Kosberg, J., Kaufman, A., Burgio, L., Leeper, J., \& Sun, F. (2007). Family caregiving to those with dementia in rural Alabama: Racial similarities and differences. Journal of Aging and Health, 19, 3-21. 
Kuroda, A., Tanaka, K., Kobayashi, R., Ito, T., Ushiki, A., \& Nakamura, K. (2007). Effect of care manager support on healthrelated quality of life of caregivers of impaired elderly: One-year longitudinal study. Industrial Health, 45, 402-408.

Leon, J., \& Neumann, P. J. (1999). The cost of Alzheimer's disease in managed care: A cross-sectional study. American Journal of Managed Care, 5, 867-877.

Mittelman, M., Haley, W. E., Clay, O. J., \& Roth, D. L. (2006). Improving caregiver well-being delays nursing home placement of patients with Alzheimer disease. Neurology, 67, 1592-1599.

Morgan, D. G., Semchuk, K. M., Stewart, N. J., \& D’Arcy, C. (2002). Rural families caring for a relative with dementia: Barriers to use of formal services. Social Science \& Medicine, 55, 1129-1142.

Nichols, P. R. (2001). Alzheimer's disease caregivers: Personal conflict and depression. Dissertation Abstracts International: Section B. Sciences and Engineering, 61, 6715.

Oppo, A. (1990). "Where there's no woman there's no home": Profile of the agro-pastoral family in nineteenth-century Sardinia. Journal of Family History, 15, 483-502.

Oppo, A. (1991). Madri, figlie e sorelle: Solidarieta' parentali in Sardegna [Mothers, daughters and sisters: Parental solidarity in Sardinia]. Polis, April, 21-48.

Organization for Economic Co-operation and Development. (2009). OECD rural policy review: Italy. Paris, France: Author.

Ostwald, S. K., Hepburn, K. W., Caron, W., Burns, T., \& Mantell, R. (1999). Reducing caregiver burden: A randomized psychoeducational intervention for caregivers of persons with dementia. The Gerontologist, 39, 299-309.

Rimmer, E., Wojciechowska, M., Stave, C., Sganga, A., \& O'Connell, B. (2005). Implications of the Facing Dementia Survey for the general population, patients and caregivers across Europe. International Journal of Clinical Practice Supplement, 59(146), 17-24.

Rinaldi, P., Spazzafumo, L., Mastriforti, R., Mattioli, P., Marvardi, M., Polidori, M. C., . . . Mecocci, P. (2005). Predictors of high level of burden and distress in caregivers of demented patients: Results of an Italian multicenter study. International Journal of Geriatric Psychiatry, 20, 168-174.

Rothera, I., Jones, R., Harwood, R., Avery, A. J., Fisher, K., James, V., ... Waite, J. (2008). An evaluation of a specialist multiagency home support service for older people with dementia using qualitative methods. International Journal of Geriatric Psychiatry, 23, 65-72.

Sanders, S. (2005). Is the glass half empty or full? Reflections on strain and gain in caregivers of individuals with Alzheimer's disease. Social Work in Health Care, 40, 57-73.

Sansoni, J., Vellone, E., \& Piras, G. (2004). Anxiety and depression in community-dwelling Italian Alzheimer's disease caregivers. International Journal of Nursing Practice, 10, 93-100.

Scott, N. W., Fayers, P. M., Aaronson, N. K., Bottomley, A., de Graeff, A., Groenvold, M., . . . Sprangers, M. A. on behalf of the EORTC Quality of Life Group and the Quality of Life CrossCultural Meta-Analysis Group. (2008). The relationship between overall quality of life and its subdimensions was influenced by culture: Analysis of an international database. Journal of Clinical Epidemiology, 61, 788-795.

Serrano-Aguilar, P. G., Lopez-Bastida, J., \& Yanes-Lopez, V. (2006). Impact on health-related quality of life and perceived burden of informal caregivers of individuals with Alzheimer's disease. Neuroepidemiology, 27, 136-142.

Smith, B., Chur-Hansen, A., Neale, A., \& Symon, J. (2008). Quality of life and cholinesterase inhibitors: A qualitative study of patients with Alzheimer's disease and their carers. Australasian Psychiatry, 16, 433-437.

Smith, S. A., \& Bell, P. A. (2005). Examining the effectiveness of the Savvy Caregiver Program among rural Colorado residents. Rural and Remote Health, 5, 466.

Smith, S. C., Murray, J., Banerjee, S., Foley, B., Cook, J. C., Lamping, D. L., . . Mann, A. (2005). What constitutes healthrelated quality of life in dementia? Development of a conceptual framework for people with dementia and their carers. International Journal of Geriatric Psychiatry, 20, 889-895.

Sorge, A. (2007). The free highlands: Honor, identity, and changes in central Sardinia. Dissertation Abstracts International: Section A. Humanities and Social Sciences, 68, 1531.

Spadin, P. (2008). Il caregiver del malato di Alzheimer [The caregiver of a person with Alzheimer's disease]. Giornale Italiano di Medicina del Lavoro ed Ergonomia, 30, B15-B21.

Steadman, P., Tremont, G., \& Davis, J. (2007). Premorbid relationship satisfaction and caregiver burden in dementia caregivers. Journal of Geriatric Psychiatry and Neurology, 20, 115-119.

Tommis, Y., Seddon, D., Woods, B., Robinson, C. A., Reeves, C., \& Russell, I. T. (2007). Rural-urban differences in the effects on mental well-being of caring for people with stroke or dementia. Aging \& Mental Health, 11, 743-750.

Vellone, E., \& Piras, G. (2009). Il caregiving a persone anziane in Sardegna [Caregiving to elderly people in Sardinia]. Infermiere Oggi, 19, 3-19.

Vellone, E., Piras, G., Talucci, C., \& Cohen, M. Z. (2008). Quality of life for caregivers of people with Alzheimer's disease. Journal of Advanced Nursing, 61, 222-231.

Vellone, E., Piras, G., Venturini, G., \& Alvaro, R. (2010). Positive and negative impact in caring for older people living in Sardinia, Italy. Journal of Clinical Nursing, 19(Suppl. 1), 131.

Vellone, E., Sansoni, J., \& Cohen, M. Z. (2002). The experiences of Italians caring for family members with Alzheimer's disease. Journal of Nursing Scholarship, 34, 323-329.

Warner, J. A. (1999). Perceptions of family caregivers of noninstitutionalized Alzheimer's patients about support groups. Dissertation Abstracts International: Section A. Humanities and Social Sciences, 60, 845.

Whitehouse, P. J., Gaines, A. D., Lindstrom, H., \& Graham, J. E. (2005). Anthropological contributions to the understanding of agerelated cognitive impairment. Lancet Neurology, 4, 320-326.

Wilks, S. E., \& Croom, B. (2008). Perceived stress and resilience in Alzheimer's disease caregivers: Testing moderation and mediation models of social support. Aging \& Mental Health, 12, 357-365. 